\title{
Research and Development of Virtual Reality Somatosensory Game based on Unity3D and Exercise Bike
}

\author{
Xufeng Ma ${ }^{1,2}$, Junghyen Kim ${ }^{1}$, Joonki Paik ${ }^{1 *}$ \\ 1. Graduate School of Advanced Imaging Science, Multimedia and Film, Chung-Ang University, 06974, Korea, \\ 2. Qingdao University of Science and Technology, 266061, China
}

\begin{abstract}
Research and development of virtual reality somatosensory game based on Unity3D and exercise bike is conducted in this manuscript. The novelty of the paper is focused on: (1) The game design method is used to realize a game design method based on the exercise bike platform, which is versatile and can be applied to other simulation interactive systems. And try to solve the rigid exercise of sports games, make the exercises vivid and interesting, give full play to the entertainment characteristics of electronic games, and let participants get a virtual and real experience. (2) In program development, using game engine as development tool improves the reuse of code, reduces the difficulty of development and shortens the development cycle. The key technologies of network communication, collision detection and artificial intelligence are studied and applied in fitness bicycle somatosensory game. The performance of the proposed model is validated through the experimental analysis. In the future, the applications will be tested.
\end{abstract}

Keywords:- Virtual reality, somatosensory game, Unity3D, exercise bike.

\section{INTRODUCTION}

The charm of virtual reality technology is to bring the users into a virtual but very real world, while making it difficult to distinguish true from false. With the update and iteration of information technology, virtual reality technology has played an important role in medical, military, political, real estate, transportation, urban planning and other fields. Based on the literature reivew, applications can be summarized as follows.

The characteristics of entertainment industry determine the inevitable advantages of interactivity and immersion. The realistic interactivity of general virtual reality provides the entertainment industry with a new and unprecedented way of experience. The movie can be viewed on VR glasses. With the improvement of the virtual reality technology and network transmission technology, VR has strengthened the technical support of general online and offline entertainment services, optimized the experience of games, live streaming, movies and other services, and enhanced the audience's participation and immersion experience [1-4].
Virtual reality technology is applied in urban planning, interior design, real estate sales and other fields, which solves the uncertainty of user pertinence and design results in this kind of the industry. Interactive design, interactive browsing experience, virtual interactive assembly and collocation and other real-time visualization functions provide users with the possibility of "expected realization" to the user. Designers and users can understand the simulation effect of the product before the construction of the product [5-8].

$>$ The current mainstream VR headset manufacturers all regard gaming applications as the most important business development focus of virtual reality at this stage. The similarity between the video game industry and virtual reality is very high, and the application of VR in the game industry has a huge market prospect. At present, game manufacturers are the most spared in terms of VR layout, either cooperating with VR equipment vendors or developing VR equipment and game content independently [9-11].

The design process of popular science games based on virtual reality is shown in Figure 1, which is divided into three stages: design, development, and the application. The design stage includes concept creativity, game background setting, game plot design, game task design, game level design, etc. This part of the work is jointly discussed by the entire game team, and game planners write game design ideas into game scripts, which is convenient The game team understands the various components of the game and the decomposition of the game, and formulates a development plan, which focuses on the integration of game content and teaching content. In the next sections, we will discuss the proposed method in detail. 


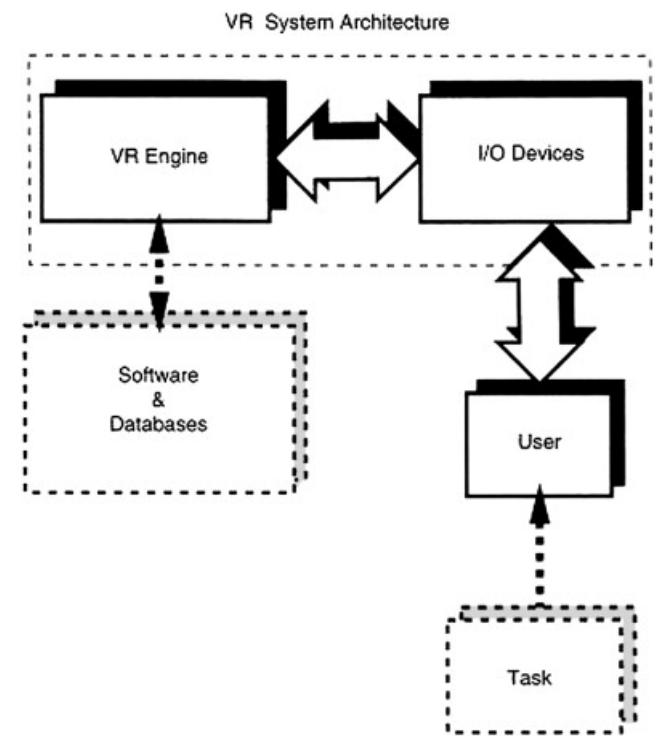

Fig 1:- Architecture of the VR System

\section{SOMATOSENSORY GAME OVERVIEW}

Somatosensory technology has only been a few years since its popularization, but it has been quite fruitful in both technology and application. Based on the collation of existing related literature, the research on realization of somatosensory technology mainly focuses on the exploration of the sensing depth and accuracy. In the figure 2 , we present the devices for the mentioned technology [12$15]$.

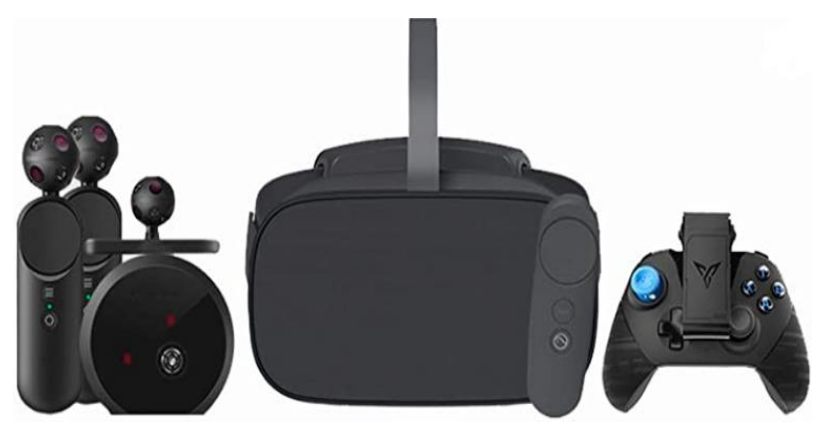

Fig 2:- Devices Used for the Mentioned Technology

The motion-sensing game connects family members with sports and the healthy entertainment, realizes the emotional exchange of many people, achieves the common sense of pleasure, and creates a relaxed and happy atmosphere of family internal affection communication. With the continuous progress of general technology, motion-sensing interactive technology will bring a big innovation for the same type of fitness games. By bringing more fun in the process of fitness, players will be able to enjoy the fun brought by sports as much as possible while realizing the purpose of exercising and losing weight successfully.

In the mainsteam systems, the technologies can be well summarized as the following aspects.
Gravity sensors, gyroscopes and magnetic sensors are used to sense the physical parameters of the user's body movements (acceleration, angular velocity, magnetic field), and then various spatial movements of the user are obtained based on these parameters.

$>$ The handle and the camera are integrated. The handle contains a gravity sensor, a gyroscope and a magnetic sensor. The camera is used to capture images of the human body. Combine these two sensors to then detect the movement and rotation of the human hand.

$>$ Obtain human body images through optical sensors (usually cameras), and use complex calculations to determine human body movements, and then interact with the content of the game.

Similar to human eyes subconsciously focusing on moving objects, Kinect will automatically find moving objects in the image that are more likely to be the human bodies, and then perform pixel-level evaluation of the depth of field image to identify different parts of the human body. In this process, the system uses an optimization algorithm to shorten the response time, uses a segmentation strategy to distinguish the human body from the background environment, and extracts useful signals from the noise. Kinect can actively track up to two players, or passively track the body and position of up to four players. At this stage, the system creates a mask in the depth-of-field image for each tracked human body, and removes background objects (such as chairs and furniture). In the figure 3, we present the platforms [16].

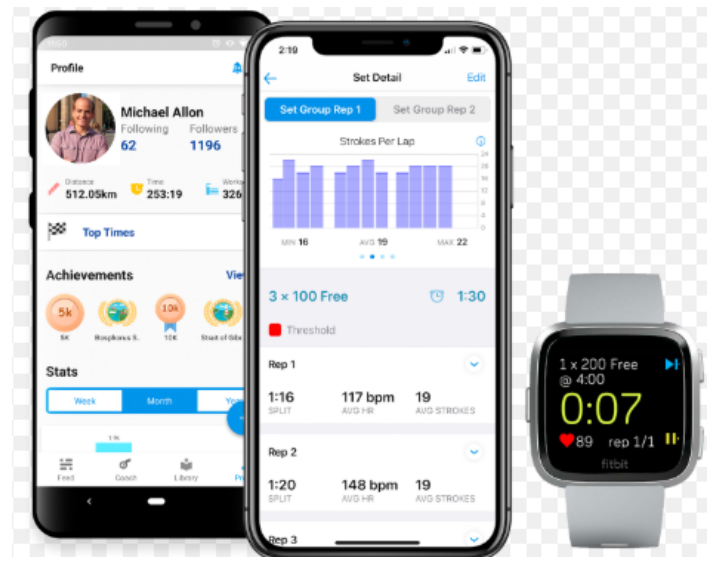

Fig 3:- Somatosensory Game Platforms

\section{UNITY3D AND EXERCISE BIKE IMPLEMENTATIONS}

Three-dimensional computer animation is mainly used to construct the model of three-dimensional body by computer, and is completely controlled by virtual camera, model, virtual light source and so on. Because of the specific characteristics of 3D animation, it is widely used in courseware making and computer special effects. It will also be used in the expression of some mechanical parts, the anatomy of human organs and the display of molecular structure, the effect is very obvious. 
In the design process, we follow the listed regulations. To realize the gameplay of the game, rules are essential. The rules defined in different games are also different, mainly including two aspects. One is its own game rules, such as the tools of Go, how to play the Go, and the outcome of Go. The second is the hidden rules that players naturally form in the game. For example, the professional ethics and discipline of the Go players. In addition, some unspoken rules have not been written into the rules one by one, relying on the players' conscious compliance.

$>$ As an emerging entertainment category, online games are similar in that online games have their own rules. The official website usually publishes game guides for novices, including game installation, game strategies, game tasks, etc., usually online as the game will use some vivid dynamic pages to demonstrate the rules in the game for players.

$>$ Some games have great success from the time they hit the market, some have fatal flaws from the start, some are obvious, but some are not immediately visible. A game that appears to have good graphics, sound, and playability loses its market due to player imbalance in the game. The rules of the game ensure that all players are equal in the game, but equality in the rules does not mean balance in the game. Only the game has the balance can greatly stimulate the interest of players, to achieve the game.

$>$ To structure gameplay in the game design process, we must first focus on the pre-game design. Game planning documents that contain gameplay build the skeleton for the overall design of the game. The game design is carried out strictly in accordance with the content of the game planning document. In the appropriate part of the game planning document, gameplay ideas are injected from details, which is conducive to game designers to improve gameplay in game production. This article refers to the content of the game playability in the last part of the game's feature set in the template of the game planning document in Chapter 2, Game Planning Overview, and puts the gameplay as a feature in the game planning.

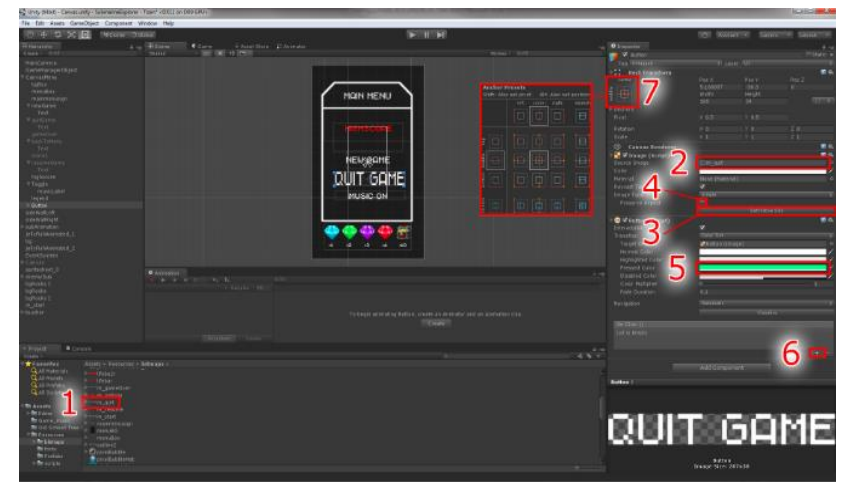

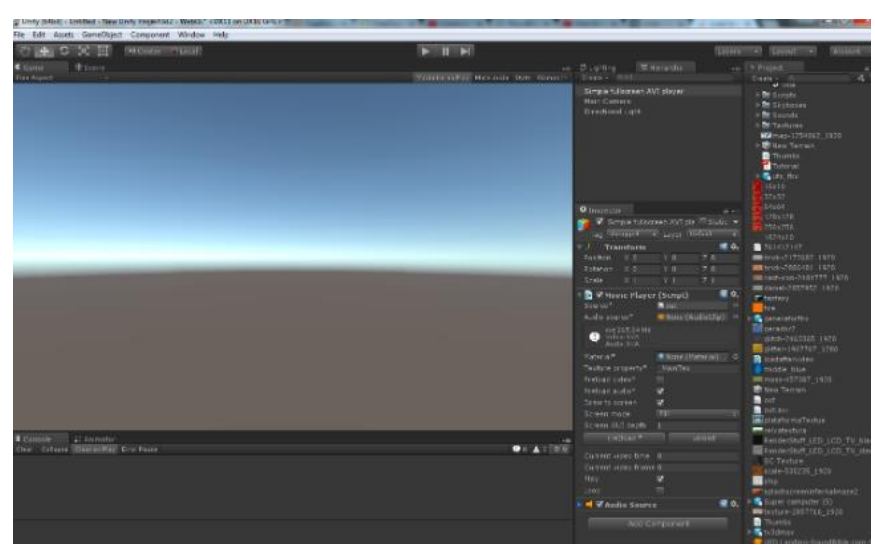

Fig 4:- Systematic Implementations

\section{CONCLUSION AND FUTURE WORK}

Research and development of virtual reality somatosensory game based on Unity3D and exercise bike is conducted in this manuscript. At present, the game industry is a hot industry, especially with the development of hightech, somatosensory games as one of the classifications use the most natural method for human-computer interaction, so that players can experience a more immersive experience. The sensation of the environment, the way to exercise the body, brain, and also the behavior at the same time while playing games and also entertainment is becoming more and more popular. Hence, this paper presents the novel perspectives of the game design. In the future, we will apply the proposed model into real applications to validate the performance.

\section{REFERENCES}

[1]. El Beheiry, M., Doutreligne, S., Caporal, C., Ostertag, C., Dahan, M. and Masson, J.B., 2019. Virtual reality: beyond visualization. Journal of molecular biology, 431(7), pp.1315-1321.

[2]. Riva, G., Wiederhold, B.K. and Mantovani, F., 2019. Neuroscience of virtual reality: from virtual exposure to embodied medicine. Cyberpsychology, Behavior, and Social Networking, 22(1), pp.82-96.

[3]. Pizzi, G., Scarpi, D., Pichierri, M. and Vannucci, V., 2019. Virtual reality, real reactions?: Comparing consumers' perceptions and shopping orientation across physical and virtual-reality retail stores. Computers in Human Behavior, 96, pp.1-12.

[4]. Farah, M.F., Ramadan, Z.B. and Harb, D.H., 2019. The examination of virtual reality at the intersection of consumer experience, shopping journey and physical retailing. Journal of Retailing and Consumer Services, 48, pp.136-143.

[5]. Sutcliffe, A.G., Poullis, C., Gregoriades, A., Katsouri, I., Tzanavari, A. and Herakleous, K., 2019. Reflecting on the design process for virtual reality applications. International Journal of Human-Computer Interaction, 35(2), pp.168-179. 
[6]. Tardif, N., Therrien, C.É. and Bouchard, S., 2019. Reexamining psychological mechanisms underlying virtual reality-based exposure for spider phobia. Cyberpsychology, Behavior, and Social Networking, 22(1), pp.39-45.

[7]. Meyer, O.A., Omdahl, M.K. and Makransky, G., 2019. Investigating the effect of pre-training when learning through immersive virtual reality and video: A media and methods experiment. Computers \& Education, 140, p.103603.

[8]. Arcioni, B., Palmisano, S., Apthorp, D. and Kim, J., 2019. Postural stability predicts the likelihood of cybersickness in active HMD-based virtual reality. Displays, 58, pp.3-11.

[9]. Kim, H.G., Lim, H.T. and Ro, Y.M., 2019. Deep virtual reality image quality assessment with human perception guider for omnidirectional image. IEEE Transactions on Circuits and Systems for Video Technology, 30(4), pp.917-928.

[10]. Ergan, S., Radwan, A., Zou, Z., Tseng, H.A. and Han, X., 2019. Quantifying human experience in architectural spaces with integrated virtual reality and body sensor networks. Journal of Computing in Civil Engineering, 33(2), p.04018062.

[11]. Lopes, D.S. and Jorge, J.A., 2019. Extending medical interfaces towards virtual reality and augmented reality. Annals of Medicine, 51(sup1), pp.29-29.

[12]. Löcken, A., Golling, C. and Riener, A., 2019, September. How should automated vehicles interact with pedestrians? A comparative analysis of interaction concepts in virtual reality. In Proceedings of the 11th International Conference on Automotive User Interfaces and Interactive Vehicular Applications (pp. 262-274).

[13]. Dias, P., Silva, R., Amorim, P., Lains, J., Roque, E., Serôdio, I., Pereira, F., Santos, B.S. and Potel, M., 2019. Using virtual reality to increase motivation in poststroke rehabilitation. IEEE computer graphics and applications, 39(1), pp.64-70.

[14]. Chen, Q., Zhang, G., Yang, X., Li, S., Li, Y. and Wang, H.H., 2018. Single image shadow detection and removal based on feature fusion and multiple dictionary learning. Multimedia Tools and Applications, 77(14), pp.18601-18624.

[15]. Loucks, L., Yasinski, C., Norrholm, S.D., MaplesKeller, J., Post, L., Zwiebach, L., Fiorillo, D., Goodlin, M., Jovanovic, T., Rizzo, A.A. and Rothbaum, B.O., 2019. You can do that?!: Feasibility of virtual reality exposure therapy in the treatment of PTSD due to military sexual trauma. Journal of anxiety disorders, 61, pp.55-63.

[16]. Deng, W., Hu, D., Xu, S., Liu, X., Zhao, J., Chen, Q., Liu, J., Zhang, Z., Jiang, W., Ma, L. and Hong, X., 2019. The efficacy of virtual reality exposure therapy for PTSD symptoms: A systematic review and metaanalysis. Journal of affective disorders, 257, pp.698709. 